\title{
Paclitaxel- and/or cisplatin-induced ocular neurotoxicity: a case report and literature review
}

This article was published in the following Dove Press journal:

OncoTargets and Therapy

31 July 2014

Number of times this article has been viewed

Ying Li*

Yanping Li*

Junyu Li

Guoliang Pi

Wenyong Tan

Department of Radiation Oncology,

Hubei Cancer Hospital, Wuhan,

People's Republic of China

*These authors contributed equally to this work
Correspondence: Wenyong Tan

Department of Radiation Oncology,

Hubei Cancer Hospital, II 6 South Road,

Zhuodaoquan, Wuhan 430079,

People's Republic of China

Tel +86 288767 I 353

Fax +862887670469

Email tanwyym@hotmail.com
Abstract: Paclitaxel (PTX) and/or cisplatin (CDDP), as important cytotoxic anti-cancer agents, are widely used to treat various solid tumors. Both may cause moderate or severe neurotoxicity, but ocular neurotoxicity is also occasionally reported. A patient diagnosed with nasopharyngeal cancer suffering acute ocular neurotoxicity 10 days after paclitaxel and CDDP administration at the recommended dose is described in the present case report, and PTX- and/or CDDP-induced ocular neurotoxicity are summarized according to previous reports. Possible mechanisms and the potential diagnostic, therapeutic and predictive strategies of PTX- and/or CDDP-induced ocular neurotoxicity are reviewed, to help the oncologist to take the infrequent toxicity of cytotoxic drugs into account and improve patient safety during anti-cancer therapy.

Keywords: cytotoxic drugs, PTX, CDDP, oncology, chemotherapy

\section{Introduction}

Paclitaxel (PTX) + cisplatin (CDDP) or PTX + carboplatin have frequently been used in combination to treat various solid tumors. Though the toxicities inevitably increased, the toxicitic profile usually remained unchanged. Rare and severe toxicities, such as ocular neurotoxicity, warrant more attention due to their negative impact on the quality of life of patients who received various anti-cancer therapies. PTX- and/or CDDPinduced ocular neurotoxicity (PCION) have rarely been reported in the literature. ${ }^{1-4}$ Herein, we describe a patient with nasopharyngeal cancer who suffered PCION after chemoradiotherapy, and review the clinical manifestations, diagnostic and therapeutic strategies, and the possible molecular mechanisms which improve patient safety during anti-cancer therapy.

\section{Case presentation}

The patient provided written informed consent for this case report. A 56-year-old male patient was diagnosed pathologically with a low-differentiated non-keratinized squamous cell via a nasopharyngeal biopsy performed using electronic nasopharyngeal endoscopy. The primary tumor had invaded locally, including the cervical lymph nodes. Based on the 2010 American Joint Committee on Cancer staging system, the tumor was stage III with T2N2M0. Intensity-modulated radiotherapy (IMRT) was delivered at three doses from July to August 2013 according to our departmental protocol (Department of Radiation Oncology, Hubei Cancer Hospital, Wuhan, People's Republic of China). ${ }^{5}$ The radiation dose to gross primary tumor and positive lymph nodes in the neck, high-risk region, and low-risk elective nodal region were $70 \mathrm{~Gy}$, 
$60.3 \mathrm{~Gy}$, and $54 \mathrm{~Gy}$, respectively. IMRT was delivered with 30 daily fractions in 6 weeks (five fractions per week). The maximal radiation dose to the optic chiasm and the left and right optic nerves were 32.1 Gy, 16.7 Gy, and 17.7 Gy, respectively (Figure 1). Complete response was confirmed by both magnetic resonance imaging (MRI) and electronic nasopharyngeal endoscope 2 months after completing radiotherapy. In addition, patient also has well controlled primary hypertension and chronic hepatitis B.

Based on our institutional guidelines, the patient was administered adjuvant chemotherapy comprising PTX (150 mg/m²) (Harbin Pharmaceutical Group Bioengineering Co, Ltd, Harbin, People's Republic of China) and CDDP (75 mg/m²) (Supertrack Biopharmaceutical Co, Ltd, Yunnan, People's Republic of China) on the day immediately following the post-radiotherapy evaluation. During the initial chemotherapy, he suffered grade 2 gastrointestinal (nausea and vomiting) and grade 4 hematological (neutropenia) toxicity, as described by the Common Terminology Criteria for Adverse Events Version $4.0,{ }^{6}$ but he recovered after receiving antiemetic and granulocyte-colony stimulating factor medications.

Ten days after the chemotherapeutic drugs were administered, he complained of severe headache, nausea, and vomiting. Fourteen hours after his first complaint, he reported blurred vision with transitory lightning scotoma in the left eye and right hand numbness. After 22 hours, he gradually lost central vision in the left eye. A detailed physical examination, complete blood count, liver and renal biochemistry, blood glucose, and serum electrolyte concentration were measured and all the result showed no clinical abnormality. His vital signs including body
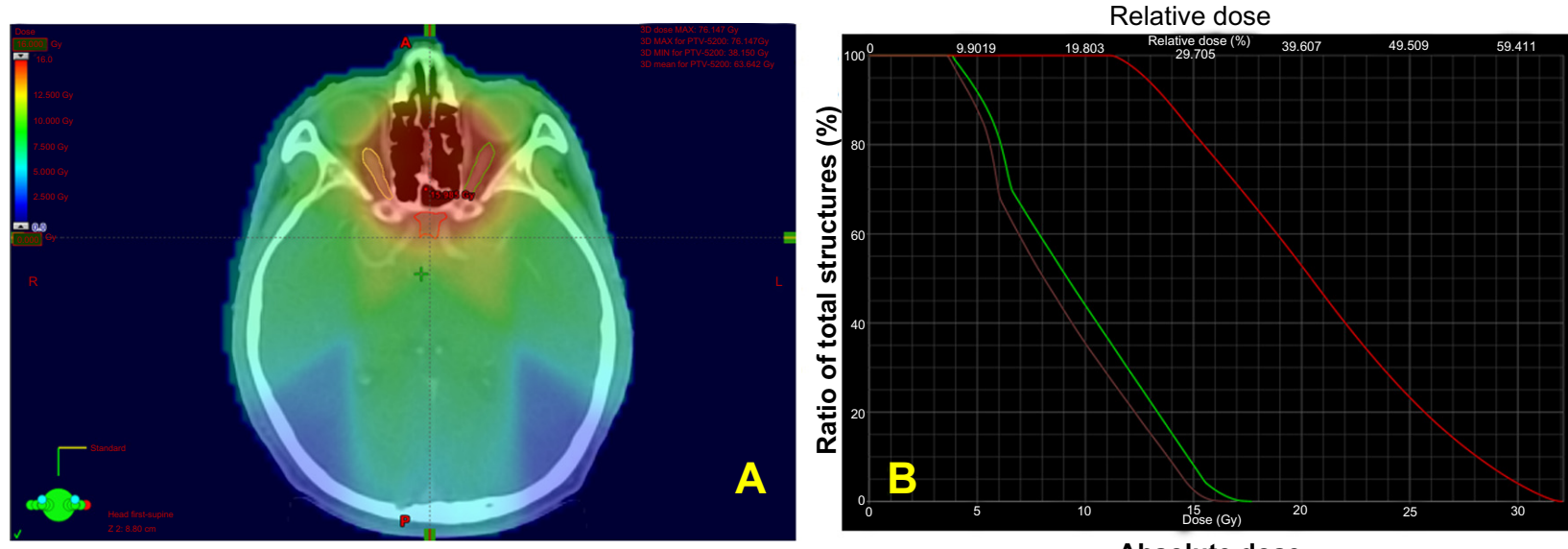

Absolute dose temperature, pulse rate, blood pressure, and respiratory rate were of normal, and no other significant physical signs were found when the patient was cooperative, conscious, and reasonably fluent during the examinations. Computed tomography (CT) and MRI were performed immediately, but there was no significant tumor recurrence or radiationassociated damage observed.

Several ophthalmic examinations were performed after the patient became blind; the best-corrected visual acuities were 0.3 on the right and only slight light perception on the left. The intraocular pressures were normal bilaterally at $11 \mathrm{mmHg}$ on the left and $13 \mathrm{mmHg}$ on the right. The pattern visual-evoked potentials (VEPs) and transient, flash electroretinograms (ERGs) were abnormal (Figure 2A and C). Non-specific waves were found in his left eye (Figure 2A and $\mathrm{C}$ ), but the VEP and ERG were normal in his right eye (Figure 2B and C). The direct ophthalmoscopic examination of the fundus, retina, and vitreous humor was normal bilaterally. The right eye had a normal visual field; the left eye visual field could not be assessed due to its loss of light perception (Figure 3). A temporal hemianopsia, and a nasal peripheral visual field defect were found in the right eye (Figure 3).

Two days after losing his left-sided vision, hypopsia appeared in the right eye, and 72 hours later, he developed complete blindness bilaterally. Numerous therapies were administered, including glucocorticoids (methylprednisolone), calcium antagonists (flunarizine), vasodilators, and neuro-nutrition drugs over the following 2 weeks, but his sight did not improve in the 6 months following adjuvant chemotherapy, and he had a very poor quality of life.

Figure I Color wash and dose-volume histogram.

Note: The radiation dose distribution at the optic chiasm (red solid line), and the left (green solid line) and right (purple solid line) optic nerves are shown in a color wash (A) and dose-volume histogram (B). 

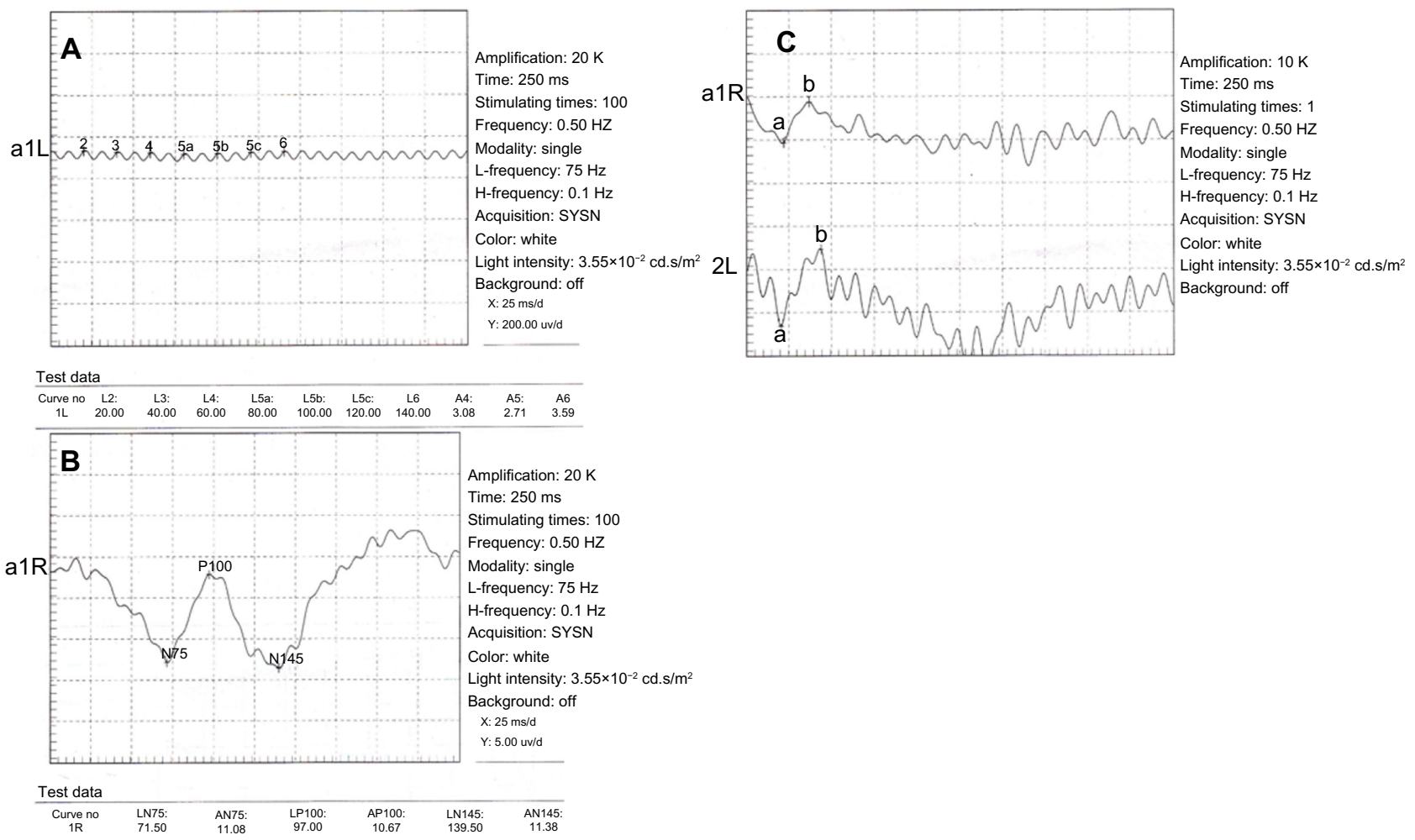

Figure 2 Flash visual-evoked potential and electroretinogram results.

Notes: During flash visual-evoked potential (A and $\mathbf{B})$, and electroretinogram (C), there was no waveform observed in the left eye (A and $\mathbf{C})$, but a normal wave was found in the right (B and $\mathbf{C}$ ). SYSN means the synchronic signal was used during the information acquisition. N75, PI00 and NI45 are three different waves in pattern visual-evoked potential.

Abbreviations: L-frequency, low frequency; H-frequency, high frequency; SYSN, synchronous signal.

\section{Discussion}

Anti-cancer drug-induced ocular neurotoxicity is extremely rare. Some of these toxicities are irreversible and might worsen the patient's quality of life, or even cause patient disability. In the following, we will discuss the clinical manifestation, possible pathogenesis, diagnosis, and therapeutic strategies against PCION.

\section{Clinical manifestations}

Chemotherapeutic agent-induced ocular neurotoxicity is commonly associated with interferon. ${ }^{7}$ The main clinical manifestations have been described previously ${ }^{4,8-16}$ and are summarized in Table 1. Ocular neurotoxicity generally presents as 1) blepharitis and conjunctivitis; 2) hemianopia, homonymous or bi-temporal visual field defects; 3 ) periorbital edema with firm eyelid swelling and ocular pain; 4) retinal toxicity with maculopathy characterized by pigmentary changes resulting from localized retinal pigment disturbances, altered color perception attributable to cone dysfunction, or mild retinal ischemic changes such as cotton-wool spots and posterior pole intraretinal hemorrhage; and 5) some other rare ocular toxicities.
Cisplatin-associated neurotoxicity is dose-dependent. ${ }^{17}$ Although it occurs infrequently, the previous studies indicated that visual impairment may occur secondary to CDDP-related neurotoxicity. ${ }^{17}$ The severe neurotoxicity was extremely uncommon at a cumulative dose below $400 \mathrm{mg} / \mathrm{m}^{2}$, but the incidence increased at a $600-800 \mathrm{mg} / \mathrm{m}^{2}$ cumulative dose. ${ }^{17}$ Children, young patients, and those with renal dysfunction seem to be the highest risk groups for cisplatin-related neurotoxicity. ${ }^{7}$ The visual alterations might also result from the optic neuritis and cortical blindness, which may be accompanied by seizure activity. ${ }^{7,18}$ Wang et a ${ }^{11}$ reported that occlusion of a retinal artery branch and bilateral blindness is associated with high-dose cytotoxic agents such as carmustine and CDDP. In a report by Kwan et al, ${ }^{4}$ severe macular ischemia and retinal neovascularization occurred in a patient who received CDDP-contained chemotherapy.

Paclitaxel-induced neurotoxicity has been reported previously. ${ }^{19}$ The patients typically complained of a transient and scintillating scotoma, visual impairment, or bilateral cystoid macular edema. The majority of these symptoms occurred within the first 30 minutes of drug administration and completely resolved within 3 hours. These symptoms 
Fixation monitor: off Fixation target: central Fixation losses: $\mathrm{O} / \mathrm{O}$ False POS errors: $6 \%$ False NEG errors: $0 \%$

Test duration: 08:03

Fovea: off

$\begin{array}{lll}\text { Stimulus: III, white } & \text { Pupil diameter: } & \text { Date: 10-29-2013 } \\ \text { Background: } 31.5 \text { ASB Visual acuity: } & \text { Time: 11:11 am } \\ \text { Strategy: SITA-fast } & \text { RX: DS DC x } & \text { Age: } 56\end{array}$

Strategy: SITA-fast
Age: 56

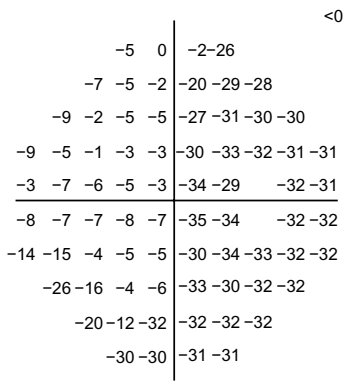

Total deviation

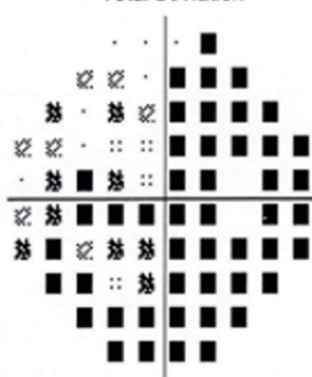

\section{:: $<5 \%$}

2. $<2 \%$

$<1 \%$

[ $<0.5 \%$

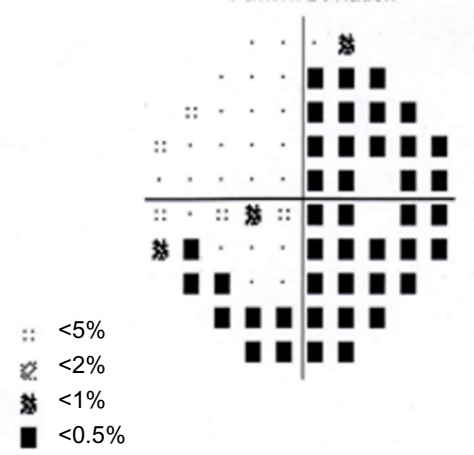

Figure 3 Visual field test.

Note: Temporal hemianopsia and a nasal peripheral visual field defect were diagnosed during the right eye visual field study.

Abbreviations: O/O, 0\%; POS, positive; NEG, negative; SITA-fast, Swedish Interactive Threshold Algorithms; RX, correction of refractive errors; DS, Diopter sphere; DC, Diopter cylinder; GHT, Glaucoma Hemifield Test; VFI, visual field index; MD, mean defect; PSD, pattern standard deviation.

Table I Summarized paclitaxel- and/or cisplatin-induced ocular toxicity reported in prior and present studies

\begin{tabular}{|c|c|c|c|c|}
\hline$\overline{\text { Author(s) }}$ & Case number & Toxicity & Drug & Diagnosis \\
\hline$\overline{\text { Kwan et al }{ }^{4}}$ & $\mathrm{I}$ & Hemianopia & CDDP & Nonseminomatous germ cell testicular tumor \\
\hline Berman and Mann ${ }^{8}$ & I & Cortical blindness & CDDP & Embryonic cell carcinoma of the testicle \\
\hline Wilding et $\mathrm{al}^{9}$ & 13 & Blurred vision & CDDP & Ovarian carcinoma \\
\hline Tan and Walsh ${ }^{10}$ & 2 & Photopsia & CDDP, PTX & Lung cancer \\
\hline Wang et al" & I & Bilateral blindness & Carmustine, CDDP & Breast carcinoma \\
\hline Watanabe et $\mathrm{al}^{12}$ & I & Visual disturbance & Carboplatin & Glioblastoma \\
\hline Wu et $a^{13}$ & I & Intraorbital and intraocular pain & CDDP & Glioblastoma multiforme \\
\hline Scaioli et al ${ }^{14}$ & I & Optic neuropathy & PTX, doxorubicin & Breast cancer \\
\hline Modi and Dubovy ${ }^{15}$ & I & Maculopathy & PTX & Breast cancer \\
\hline Joshi and Garretson ${ }^{16}$ & I & Cystoid macular edema & PTX & Breast cancer \\
\hline Li et al (present study) & I & Bilateral blindness & CDDP, PTX & Nasopharyngeal cancer \\
\hline
\end{tabular}

Abbreviations: PTX, paclitaxel; CDDP, cisplatin. 
occurred more frequently in patients who received doses greater than $250 \mathrm{mg} / \mathrm{m}^{2} .{ }^{20,21}$ Tan et al ${ }^{10}$ described two patients with breast and ovarian cancer who suffered ocular toxicity after the administration of PTX and carboplatin. Though the underlying causes remained unknown, the authors surmised that PTX was the primary agent. Bakbak et $\mathrm{al}^{22}$ assessed CDDP- and PTX-associated toxicities in the optic nerve by measuring the retinal nerve fiber layer (RNFL) thickness and visual field changes in patients with lung cancer who received systemic CDDP and PTX. They found the peripapillary RNFL thicknesses and visual field indices changed based on frequency-doubling technology (FDT) perimetry.

\section{Possible mechanisms}

The mechanism of ocular neurotoxicity remains unknown, and neither the ischemic nor electrophysiological hypotheses could fully explain the pathogenesis of PCION in the previous study ${ }^{23} \mathrm{~A}$ study by Scaioli et a ${ }^{24}$ suggested that the visual symptoms and electrophysiological changes following intravenous PTX administration were likely caused by retinal vascular dysregulation or optic nerve ischemia. Because the cystoid macular edema occurred after treatment of PTX, one theory is that Müller cell toxicity results subsequent to intracellular fluid accumulation and subclinical extracellular fluid leakage. In patients with reversible scotoma, the abnormal visual-evoked potential was comparable to those with changes observed in ischemic neuropathies, which suggested that the target of anti-cancer drugs such as PTX was within the optic nerve. ${ }^{19}$ Brain irradiation and/or surgery are high risk factors because cystoid macular edema can result in disruption of the normal blood-retinal barrier. ${ }^{16,24}$

Cisplatin or PTX may affect optic nerve function, and the combination of CDDP with PTX may synergistically increase the neurotoxicity risk to the optic nerve. In our case, however, the primary cause of the patient's ocular symptoms and blindness remain unknown. The cause may potentially be attributed to CDDP administration alone, PTX alone, the combination of PTX with CDDP, or the interaction of cytotoxic drugs with radiation. To investigate the exact cause of the loss of eyesight, both CT and MRI scanning of head and neck, and laboratory biochemical examination including serum electrolyte, were performed. The potential causes that could result in ocular disease were excluded by various examinations. They included the uncontrolled primary tumor, radiation-induced brain and nerve damage, the central neural and cerebrovascular disease, chemotherapeutic drug-related myelosuppression, imbalance of serum electrolyte, and so on. For the patient in our report, the possible cause of PCION included cytotoxic drugs, radiation, and the patient's concomitant diseases (hypertension and chronic hepatitis B). The possibility of both radiation treatment and concomitant diseases causing PCION was extremely low because all the concomitant diseases were well controlled. Additionally, the maximal radiation dose to the optical nerves and chiasm was $<32 \mathrm{~Gy}$, which is far below the tolerance to the radiation. Though we could not accurately ascertain the exact cause of our patient's blindness, and did not know the precise interaction of cytotoxic agent and radiation or the variation of the normal tissue's response to anti-cancer therapy during the complicated context of chemoradiotherapy, we could still infer cytotoxicity of PTX and/or CDDP as the primary cause.

\section{Strategies for detection, treatment and prediction}

Because of its scarcity, PCION was usually underestimated or considered minor when compared to other life-threatening complications. ${ }^{17}$ Oncologists should take all potential severe toxicities into consideration prior to initial treatment and be familiar with their pathogenesis and management. Despite the lack of consensus outlining routine ophthalmologic monitoring and ocular toxicity management at present, for some drugs with potential ocular toxicity a baseline ophthalmologic examination and regular monitoring is strongly recommended before treatment. The monitoring examinations could include visual acuity, tonometry, fundoscopy, color vision test, automated perimetry, retinal photography, and others. ${ }^{17}$ Potential treatment options including warm compress, eyelid hygiene, corticosteroids, topical anti-inflammatory medications and lubricants, and avoiding light exposure may prove useful, but further clinical study is needed. Hofstra et $\mathrm{al}^{25}$ reported that flunarizine, a selective calcium channel antagonist, has been used successfully to treat one patient diagnosed with visual-evoked potential abnormalities. Kwan et $\mathrm{al}^{4}$ reported a case of bilateral panretinal laser photocoagulation, but the therapy was ineffective because the best-corrected visual acuity remained unchanged 6 months later. Based on previous reports, nearly all PCIONs were irreversible, and the best-corrected visual acuity failed to improve significantly. Currently, there is no biomarker available to effectively predict these severe PCIONs.

\section{Conclusion}

PTX- and CDDP-induced ocular toxicity is extremely rare and usually irreversible. The clinical manifestations mainly include abnormal vision, hemianopia, photopsia, visual disturbance, blindness, ocular pain, cystoid macular edema, 
and other similar symptoms. The possible mechanisms of PCION might be related to the ischemic and electrophysiological changes within ocular neural structures. There are no effective strategies for the early detection, treatment, and prediction of PCION, which is warranted for further investigation. Clinical oncologists should consider the risk of severe PCION prior to initiating anti-cancer treatment.

\section{Disclosure}

The authors report no conflicts of interest in this work.

\section{References}

1. Ziske CG, Schöttker B, Gorschlüter M, et al. Acute transient encephalopathy after paclitaxel infusion: report of three cases. Ann Oncol. 2002;13(4):629-631.

2. Cavaletti G, Bogliun G, Marzorati L, et al. Peripheral neurotoxicity of taxol in patients previously treated with cisplatin. Cancer. 1995;75(5): 1141-1150.

3. Anand A, Huberman M. Peripheral neurotoxicity of taxol in patients previously treated with cisplatin. Cancer. 1995;76(5):916-917.

4. Kwan AS, Sahu A, Palexes G. Retinal ischemia with neovascularization in cisplatin related retinal toxicity. Am J Ophthalmol. 2006;141(1): 196-197.

5. Tan W, Li Y, Han G, et al. Target volume and position variations during intensity-modulated radiotherapy for patients with nasopharyngeal carcinoma. Onco Targets Ther. 2013;6:1719-1728.

6. National Cancer Institute. Common Terminology Criteria for Adverse Events (CTCAE) Version 4.0. U.S. Department of Health and Human Services. Available from: http://evs.nci.nih.gov/ftp1/CTCAE/ CTCAE_4.03_2010-06-14_QuickReference_5x7.pdf. Accessed July 11, 2014.

7. Walsh TJ, Clark AW, Parhad IM, Green WR. Neurotoxic effects of cisplatin therapy. Arch Neurol. 1982;39(11):719-720.

8. Berman IJ, Mann MP. Seizures and transient cortical blindness associated with cis-platinum (II) diamminedichloride (PDD) therapy in a thirty-year-old man. Cancer. 1980;45(4):764-766.

9. Wilding G, Caruso R, Lawrence TS, et al. Retinal toxicity after highdose cisplatin therapy. J Clin Oncol. 1985;3(12):1683-1689.

10. Tan WW, Walsh T. Ocular toxicity secondary to paclitaxel in two lung cancer patients. Med Pediatr Oncol. 1998;31(3):177.
11. Wang MY, Arnold AC, Vinters HV, Glasgow BJ. Bilateral blindness and lumbosacral myelopathy associated with high-dose carmustine and cisplatin therapy. Am J Ophthalmol. 2000;130(3):367-368.

12. Watanabe W, Kuwabara R, Nakahara T, et al. Severe ocular and orbital toxicity after intracarotid injection of carboplatin for recurrent glioblastomas. Graefes Arch Clin Exp Ophthalmol. 2002;240(12): 1033-1035.

13. Wu HM, Lee AG, Lehane DE, Chi TL, Lewis RA. Ocular and orbital complications of intraarterial cisplatin. A case report. JNeuroophthalmol. 1997;17(3):195-198.

14. Scaioli V, Caraceni A, Martini C, et al. Visual evoked potentials findings in course of paclitaxel doxorubicin combination chemotherapy. Report of a case. J Neurooncol. 1995;25(3):221-225.

15. Modi D, Dubovy SR. Non-leaking Cystoid Maculopathy Secondary to Systemic Paclitaxel. Ophthalmic Surg Lasers Imaging Retina. 2013; 44(2):183-186

16. Joshi MM, Garretson BR. Paclitaxel maculopathy. Arch Ophthalmol. 2007;125(5):709-710

17. Agustoni F, Platania M, Vitali M, et al. Emerging toxicities in the treatment of non-small cell lung cancer: ocular disorders. Cancer Treat Rev. 2014;40(1):197-203.

18. Katz BJ, Ward JH, Digre KB, Creel DJ, Mamalis N. Persistent severe visual and electroretinographic abnormalities after intravenous Cisplatin therapy. J Neuroophthalmol 2003;23(2):132-135.

19. Capri G, Munzone E, Tarenzi E, et al. Optic nerve disturbances: a new form of paclitaxel neurotoxicity. J Natl Cancer Inst. 1994;86(14): 1099-1101.

20. Seidman AD, Barrett $\mathrm{S}$, Canezo S. Photopsia during 3-hour paclitaxel administration at doses $\geq 250 \mathrm{mg} / \mathrm{m}^{2}$. J Clinical Oncol. 1994;12(8): 1741-1742.

21. Kuznetcova TI, Cech P, Herbort CP. The mystery of angiographically silent macular oedema due to taxanes. Int Ophthalmol. 2012;32(3): 299-304.

22. Bakbak B, Gedik S, Koktekir BE, et al. Assessment of ocular neurotoxicity in patients treated with systemic cancer chemotherapeutics. Cutan Ocul Toxicol. 2014;33(1):7-10.

23. Hagiwara H, Sunada Y. Mechanism of taxane neurotoxicity. Breast Cancer. 2004;11(1):82-85.

24. Scaioli V, Caraceni A, Martini C, Curzi S, Capri G, Luca G. Electrophysiological evaluation of visual pathways in paclitaxel-treated patients. J Neurooncol. 2006;77(1):79-87.

25. Hofstra LS, de Vries EG, Willemse PH. Ophthalmic toxicity following paclitaxel infusion. Ann Oncol. 1997;8(10):1053.
OncoTargets and Therapy

\section{Publish your work in this journal}

OncoTargets and Therapy is an international, peer-reviewed, open access journal focusing on the pathological basis of all cancers, potential targets for therapy and treatment protocols employed to improve the management of cancer patients. The journal also focuses on the impact of management programs and new therapeutic agents and protocols on

\section{Dovepress}

patient perspectives such as quality of life, adherence and satisfaction. The manuscript management system is completely online and includes a very quick and fair peer-review system, which is all easy to use. Visit http://www.dovepress.com/testimonials.php to read real quotes from published authors. 